\title{
Unsteady mixed convection flow of nanofluid on a rotating cone with magnetic field
}

\author{
S. Nadeem $\cdot$ S. Saleem
}

Received: 14 February 2013/ Accepted: 13 March 2013/Published online: 4 April 2013

(C) The Author(s) 2013. This article is published with open access at Springerlink.com

\begin{abstract}
In this article, we have presented the unsteady flow of a rotating nanofluid in a rotating cone in the presence of magnetic field. The highly nonlinear coupled partial differential equations are simplified with the help of suitable similarity transformations. The reduced nonlinear coupled equations are solved analytically with the help of homotopy analysis method. The physical features of the parameters of interest are discussed by plotting graphs and through tables.
\end{abstract}

Keywords Nanofluid - Mixed convection · MHD . Heat transfer $\cdot$ Series solution

\section{Introduction}

The study of flow over cone-shaped bodies is often encountered in many engineering applications. Only limited attentions have been focused to this kind of study. Mixed convection flow is another important subject which has fascinated the attention of several researchers due to its fundamental applications. Solar central receivers exposed to wind currents, electronic devices cooled by fans, nuclear reactors cooled during emergency shutdown, heat exchangers placed in a low velocity environment are some of the applications of mixed convection flow. The study of convective heat transfer in a rotating flows over a rotating cone is also very important phenomena for the thermal design of various types of equipment's such as rotating heat exchanger, spin stabilized missiles, containers of

S. Nadeem · S. Saleem $(\square)$

Quaid-I-Azam University, Islamabad, Pakistan

e-mail: salmansaleem_33@hotmail.com

S. Nadeem

e-mail: snqau@hotmail.com nuclear waste disposal and geothermal reservoirs (Tien 1960; Lin and Lin 1987). Initially, Heiring and Grosh (1963) studied the steady mixed convection from a vertical cone for $\operatorname{Pr}=0.7$. They applied similarity transformation which shows that Buoyancy parameter is the dominant dimensionless parameter that would set the three regions, specifically forced, free and mixed convection. Further, Himasekhar et al. (1989) presented the similarity solution of the mixed convection flow over a vertical rotating cone in a fluid for a wide range of Prandtl numbers. Anilkumar and Roy (2004) have investigated the self-similar solutions of an unsteady mixed convection flow over a rotating cone in a rotating viscous fluid. They found that similar solutions are only possible when angular velocity is inversely proportional to time. Boundary layer on a rotating cones, disks and axisymmetric surfaces with a concentrated heat surface has been given by Wang and Kleinstreur (1990). Nonsimilar solutions to the heat transfer in unsteady mixed convection flows from a vertical cone is presented by Ishak et al. (2010).

The study of magnetohydrodynamic flows in the presence of heat transfer in the form of either mixed convection or natural convection is important in number of technological and industrial applications (Aldoss 1996; bararnia et al. 2009). Such applications include the production of steel, aluminium, high performance super alloys or crystals. In crystal growth, the magnetic fields are used to suppress the convective motion induced by the arising strong fluxes to control the flow in the melt and consequently the crystal quality. Recently, Kakarantzas (2009) have examined the magnetohydrodynamic natural convection in a vertical cylinder cavity with sinusoidal upper wall temperature.

Recently nanofluids have attracted great interest because of reports of greatly enhanced thermal properties. These 
fluids are solid-liquid composite materials made of solid nanoparticles or nanofibers with a size of $1-100 \mathrm{~nm}$ suspended in liquid. Buongiorno (2006) and Kakaç and Pramuanjaroenkij (2009) have investigated a comprehensive survey of convective transporting nanofluids. Khan and Pop (2010) analyzed the development of the steady boundary layer flow, heat transfer and nanoparticle fraction over a stretching surface in a nanofluid. More recently, various problems about nanofluids are investigated by other researchers (Buongiorno 2006; Makinde and Aziz 2011; Hojjat et al. 2010; Bachok et al. 2010; Kakaç and Pramuanjaroenkij 2009; Khan and Pop 2010; Nadeem and Lee 2012; Nadeem and Haq 2012; Duangthongsuk and Wongwises 2007, 2008; Hojjat et al. 2010).

The objective of the present paper was to analyze the development in the unsteady mixed convection MHD rotating nanofluid flow on a rotating cone. The viscous flow equations for unsteady flow are presented along with heat and mass transfer analysis. The governing highly nonlinear coupled partial differential equations are first transformed to coupled ordinary differential equations using the similarity transformations and then solved analytically with the help of homotopy analysis method (Liao 2003, 2004, 2005, 2009; Liao and Cheung 2003; Abbasbandy 2006, 2008; Abbasbandy and Samadian 2008; Ellahi and Riaz 2010; Ellahi and Afzal 2009; Nadeem et al. 2010; Nadeem and Hussain 2009; Nadeem and Saleem 2013). The influences of different physical parameters are also presented and discussed graphically. Finally, our analytical and previous numerical results are in excellent agreement.

\section{Mathematical formulation}

We have considered an unsteady axisymmetric incompressible flow of nanofluid over a rotating cone in a rotating fluid in the presence of MHD. We have taken the rectangular curvilinear fixed coordinate system. Let $u$, $v$ and $w$ be the velocity components along $x$ (tangential), $y$ (circumferential or azimuthal) and $z$ (normal) directions, respectively. Both the fluid and the cone are in a rigid body rotation about the axis of cone with time-dependent angular velocity $\Omega$ either in some or reverse directions, due to which unsteadiness produces in the fluid flow. A constant magnetic field is applied in the normal direction, i.e., in the $z$ direction. The induced magnetic field is considered to be negligible because of small magnetic Reynolds number. Further there is no electric field. The wall temperature $T_{\mathrm{w}}$ and wall concentration $C_{\mathrm{w}}$ are constant functions. Geometry of the problem is shown in Fig. 1.

The boundary layer equations of motion, temperature and concentration in the presence of nanoparticles are

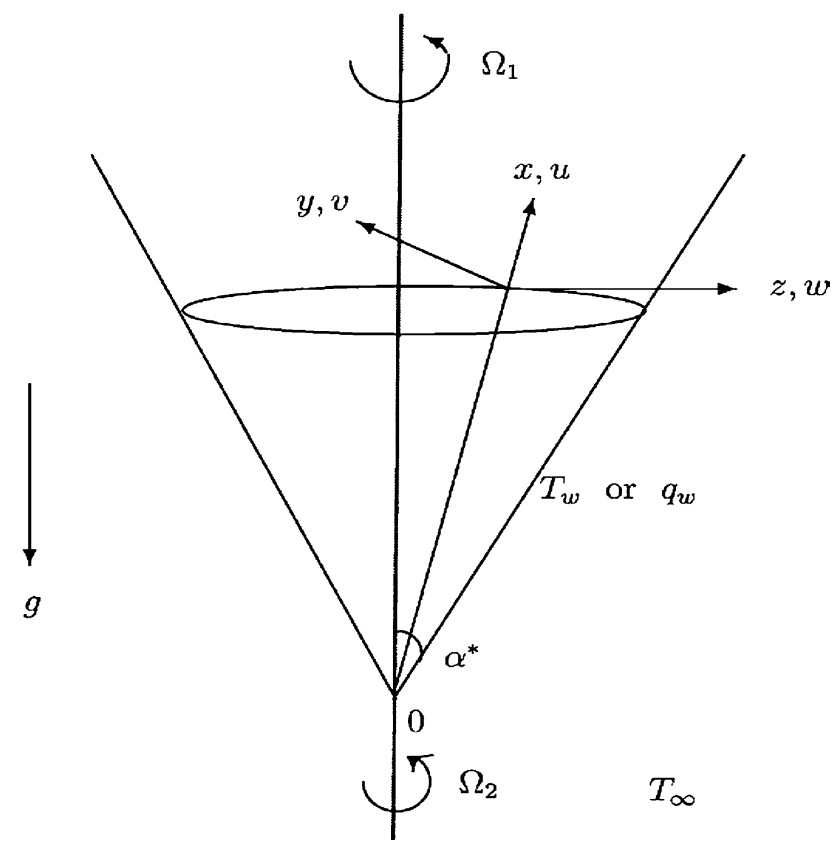

Fig. 1 Geometry of the problem

$\frac{\partial u}{\partial x}+\frac{u}{x}+\frac{\partial w}{\partial z}=0$

$\frac{\partial u}{\partial t}+u \frac{\partial u}{\partial x}+w \frac{\partial u}{\partial z}-\frac{v^{2}}{x}=-\frac{v_{e}^{2}}{x}+v \frac{\partial^{2} u}{\partial z^{2}}+g \beta \cos \alpha^{*}$

$\left(T-T_{\infty}\right)+g \beta^{*} \cos \alpha^{*}\left(C-C_{\infty}\right)-\frac{\sigma}{\rho} B^{2} u$,

$\frac{\partial v}{\partial t}+u \frac{\partial v}{\partial x}+w \frac{\partial v}{\partial z}+\frac{u v}{x}=\frac{\partial v_{e}}{\partial t}+v \frac{\partial^{2} v}{\partial z^{2}}-\frac{\sigma}{\rho} B^{2}\left(v-v_{e}\right)$,

$(\rho c)_{\mathrm{f}}\left(\frac{\partial T}{\partial t}+u \frac{\partial T}{\partial x}+w \frac{\partial T}{\partial z}\right)=\kappa \frac{\partial^{2} T}{\partial z^{2}}$

$+(\rho c)_{\mathrm{p}}\left[D_{\mathrm{B}} \frac{\partial C}{\partial z} \frac{\partial T}{\partial z}+\frac{D_{\mathrm{T}}}{T_{\infty}}\left(\frac{\partial T}{\partial z}\right)^{2}\right]$,

$\frac{\partial C}{\partial t}+u \frac{\partial C}{\partial x}+w \frac{\partial C}{\partial z}=D_{\mathrm{B}} \frac{\partial^{2} C}{\partial z^{2}}+\frac{D_{\mathrm{T}}}{T_{\infty}} \frac{\partial^{2} T}{\partial z^{2}}$,

where Eq. (1) is the continuity equation, Eqs. (2) and (3) are the momentum equations, and Eqs. (4) and (5) are the temperature and nanoparticle concentration equations. In the above equations, $k$ is the thermal diffusivity, $\alpha^{*}$ is the semivertical angle of the cone, $v$ is the kinematic viscosity, $\rho$ is the density, $\beta$ and $\beta^{*}$ are the volumetric coefficient of expansion for temperature and concentration, respectively, $C_{\infty}$ and $T_{\infty}$ are the free-stream concentration and temperature, respectively, $(\rho c)_{\mathrm{p}}$ is the nanoparticle heat capacity, $(\rho c)_{\mathrm{f}}$ is the base fluid heat capacity, $D_{\mathrm{B}}$ is the Brownian diffusion coefficient, $D_{\mathrm{T}}$ is the thermophoretic diffusion coefficient. Applying the similarity transformations and non-dimensional 
variables given in Anilkumar and Roy (2004), Eqs. (1)-(5) with the boundary conditions for prescribed wall temperature (PWT) case can be expressed as

$$
\begin{aligned}
& f^{\prime \prime \prime}-f f^{\prime \prime}+\frac{1}{2} f^{\prime 2}-2\left(g^{2}-\left(1-\alpha_{1}\right)^{2}\right)-2 \lambda_{1}(\theta+N \phi) \\
& -s\left(f^{\prime}+\frac{1}{2} \eta f^{\prime \prime}\right)-M f^{\prime}=0, \\
& g^{\prime \prime}-\left(f g^{\prime}-g f^{\prime}\right)+s\left(1-\alpha_{1}-g-\frac{1}{2} \eta g^{\prime}\right) \\
& \quad-M\left(g-1+\alpha_{1}\right)=0, \\
& \frac{1}{\operatorname{Pr}} \theta^{\prime \prime}-\left(f \theta^{\prime}-f^{\prime} \frac{\theta}{2}\right)-s\left(2 \theta+2^{-1} \eta \theta^{\prime}\right)+N b \phi^{\prime} \theta^{\prime}+N t \theta^{\prime 2}=0, \\
& \phi^{\prime \prime}-\operatorname{Le}\left\{\left(f \varphi^{\prime}-f^{\prime} \frac{\phi}{2}\right)-s\left(2 \phi+2^{-1} \eta \phi^{\prime}\right)\right\}+\frac{\mathrm{Nt}}{\mathrm{Nb}} \theta^{\prime \prime}=0 . \\
& \quad \quad \text { when } \eta=0 \\
& f(\eta)=0=f^{\prime}(\eta), g(\eta)=\alpha_{1}, \theta(\eta)=\phi(\eta)=1, \\
& f^{\prime}(\eta)=0, g(\eta)=1-\alpha_{1}, \theta(\eta)=\phi(\eta)=0, \quad \text { as } \quad \eta \rightarrow \infty .
\end{aligned}
$$

For prescribed heat flux (PHF) case, Eqs. (1)-(5) with the boundary conditions are stated as

$$
\begin{aligned}
& F^{\prime \prime \prime}-F F^{\prime \prime}+2^{-1} F^{\prime 2}-2\left(G^{2}-\left(1-\alpha_{1}\right)^{2}\right)-2 \lambda_{1}^{*}\left(\Theta+N^{*} \Phi\right) \\
& \quad-s\left(F^{\prime}+2^{-1} \eta F^{\prime \prime}\right)-M F^{\prime}=0 \\
& G^{\prime \prime}-\left(F G^{\prime}-G F^{\prime}\right)+S\left(1-\alpha_{1}-G-2^{-1} \eta G^{\prime}\right) \\
& \quad-M\left(G-1+\alpha_{1}\right)=0 \\
& \frac{1}{\operatorname{Pr}} \Theta^{\prime \prime}-\left(F \Theta^{\prime}-F^{\prime} \frac{\Theta}{2}\right)-s\left(2 \Theta+2^{-1} \eta \Theta^{\prime}\right)+\mathrm{Nb} \Phi^{\prime} \Theta^{\prime} \\
& \quad+\mathrm{Nt}^{\prime 2}=0, \\
& \Phi^{\prime \prime}-L e\left\{\left(F \Phi^{\prime}-F^{\prime} \frac{\Phi}{2}\right)-s\left(2 \Phi+2^{-1} \eta \Phi^{\prime}\right)\right\}+\frac{N t}{N b} \Theta^{\prime \prime}=0
\end{aligned}
$$

$$
\begin{aligned}
& F(\eta)=0=F^{\prime}(\eta), G(\eta)=\alpha_{1}, \Theta^{\prime}(\eta)=\Phi^{\prime}(\eta)=-1, \\
& \quad \text { when } \eta=0 \\
& F^{\prime}(\eta)=0, G(\eta)=1-\alpha_{1}, \Theta(\eta)=\Phi(\eta)=0, \text { as } \eta \rightarrow \infty
\end{aligned}
$$

where

$$
\begin{aligned}
& v_{e}=\Omega_{2} x \sin \alpha^{*}\left(1-s t^{*}\right)^{-1}, \eta=\left(\frac{\Omega \sin \alpha^{*}}{v}\right)^{\frac{1}{2}}\left(1-s t^{*}\right)^{-\frac{1}{2}} z, \\
& t^{*}=\left(\Omega \sin \alpha^{*}\right) t, u(t, x, z)=-2^{-1} \Omega x \sin \alpha^{*}\left(1-s t^{*}\right)^{-1} f^{\prime}(\eta), \\
& v(t, x, z)=\Omega x \sin \alpha^{*}\left(1-s t^{*}\right)^{-1} g(\eta), w(t, x, z) \\
& =\left(v \Omega \sin \alpha^{*}\right)^{\frac{1}{2}}\left(1-s t^{*}\right)^{-\frac{1}{2}} f(\eta), \\
& T(t, x, z)-T_{\infty}=\left(T_{\mathrm{w}}-T_{\infty}\right) \theta(\eta), T_{\mathrm{w}}-T_{\infty} \\
& =\left(T_{0}-T_{\infty}\right)\left(\frac{x}{L}\right)\left(1-s t^{*}\right)^{-2}, \\
& C(t, x, z)-C_{\infty}=\left(C_{\mathrm{w}}-C_{\infty}\right) \varphi(\eta),\left(C_{\mathrm{w}}-C_{\infty}\right) \\
& =\left(C_{0}-C_{\infty}\right)\left(\frac{x}{L}\right)\left(1-s t^{*}\right)^{-2}, \\
& \mathrm{Gr}_{1}=g \beta \cos \alpha^{*}\left(T_{0}-T_{\infty}\right) \frac{L^{3}}{v^{2}}, \operatorname{Re}_{L}=\Omega \sin \alpha^{*} \frac{L^{2}}{v}, \lambda_{1}=\frac{\mathrm{Gr}_{1}}{\mathrm{Re}_{L}^{2}} \\
& G r_{2}=g \beta^{*} \cos \alpha^{*}\left(C_{0}-C_{\infty}\right) \frac{L^{3}}{v^{2}}, \lambda_{2}=\frac{\mathrm{Gr}_{2}}{\operatorname{Re}_{L}^{2}}, N=\frac{\lambda_{2}}{\lambda_{1}} \\
& \alpha_{1}=\frac{\Omega_{1}}{\Omega}, \operatorname{Pr}=\frac{v}{\alpha}, M=\frac{\sigma B_{0}^{2}}{\rho}\left(\Omega \sin \alpha^{*}\right)^{-1}\left(1-s t^{*}\right), \\
& N^{*}=\frac{\lambda_{2}^{*}}{\lambda_{1}^{*}}, \lambda_{1}^{*}=\frac{\mathrm{Gr}_{1}^{*}}{\operatorname{Re}_{L}^{2}} \\
& \lambda_{2}^{*}=\frac{\mathrm{Gr}_{2}^{*}}{\mathrm{Re}_{L}^{2}}, \mathrm{Gr}_{1}^{*}=g \beta^{*} \cos \alpha^{*}\left(q_{0}\right) \frac{L^{4}}{\kappa v^{2}}, \\
& \mathrm{Gr}_{2}^{*}=g \beta^{*} \cos \alpha^{*}\left(\dot{m}_{0}\right) \frac{L^{4}}{\rho D v^{2}} \\
& \mathrm{Nb}=\frac{(\rho c)_{\mathrm{p}} D_{\mathrm{B}}\left(C_{\mathrm{w}}-C_{\infty}\right)}{v(\rho c)_{\mathrm{f}}}, \mathrm{Nt}=\frac{(\rho c)_{\mathrm{p}} D_{\mathrm{T}}\left(T_{\mathrm{w}}-T_{\infty}\right)}{v(\rho c)_{\mathrm{f}} T_{\infty}}, \\
& \text { Le }=\frac{v}{D_{\mathrm{B}}} .
\end{aligned}
$$

Here $\alpha_{1}$ is the ratio of angular velocity of the cone to the composite angular velocity, $\lambda_{1}$ and $\lambda_{1}^{*}$ are the buoyancy force parameter for PWT and PHF cases, respectively, $N$ and $N^{*}$ are the ratio of the Grashof numbers for PWT and PHF cases, respectively. It is zero for chemical diffusion, infinite for the thermal diffusion, positive when the buoyancy forces due to temperature and concentration difference act in the same direction and vice versa. $s$ is the unsteady parameter. The flow is accelerating if $s>0$ provided $s t^{*}<1$ and the flow is retarding, if $s<0$. Further $\alpha_{1}=0$ shows that the fluid is rotating and the cone is at rest, moreover, the fluid and the cone are rotating with equal angular velocity in the same direction for $\alpha_{1}=0.5$, and for $\alpha_{1}=1$, only the cone is in rotation. Nb, Nt, $M$ and Le denote the Brownian motion parameter, thermophoresis parameter, non-dimensional magnetic parameter and Lewis number, respectively. 
The physical quantities of interest in this problem are the local skin-friction coefficients, Nusselt number and the local Sherwood number, which are defined for the PWT case as

$$
\begin{gathered}
C_{f x}=\frac{\left[2 \mu\left(\frac{\partial u}{\partial z}\right)\right]_{z=0}}{\rho\left[\Omega x \sin \alpha^{*}\left(1-s t^{*}\right)^{-1}\right]^{2}}=-R e_{x}^{-1 / 2} f^{\prime \prime}(0), \\
C_{f y}=-\frac{\left[2 \mu\left(\frac{\partial v}{\partial z}\right)\right]_{z=0}}{\rho\left[\Omega x \sin \alpha^{*}\left(1-s t^{*}\right)^{-1}\right]^{2}}=-R e_{x}^{-1 / 2} g^{\prime}(0),
\end{gathered}
$$

or

$C_{f x} R e_{x}^{1 / 2}=-f^{\prime \prime}(0)$,

$C_{f y} R e_{x}^{1 / 2}=-g^{\prime}(0)$,

$\mathrm{Nu} R e_{x}^{-1 / 2}=-\theta^{\prime}(0)$,

$\operatorname{Sh} R e_{x}^{-1 / 2}=-\varphi^{\prime}(0)$.

\section{Solution of the problem}

The highly nonlinear coupled ordinary differential equations for PWT case will be solved analytically by homotopy analysis method (HAM). According to HAM procedure, we express $f(\eta), g(\eta), \theta(\eta)$ and $\phi(\eta)$ by a set of base functions

$\left\{\eta^{k} \exp (-n \eta) \mid k \geq 0, n \geq 0\right\}$,

in the form

$f(\eta)=a_{0,0}^{0}+\sum_{n=0}^{\infty} \sum_{k=0}^{\infty} a_{m, n}^{k} \eta^{k} \exp (-n \eta)$,

$g(\eta)=b_{0,0}^{0}+\sum_{n=0}^{\infty} \sum_{k=0}^{\infty} b_{m, n}^{k} \eta^{k} \exp (-n \eta)$

$\theta(\eta)=\sum_{n=0}^{\infty} \sum_{k=0}^{\infty} c_{m, n}^{k} \eta^{k} \exp (-n \eta)$

$\phi(\eta)=\sum_{n=0}^{\infty} \sum_{k=0}^{\infty} d_{m, n}^{k} \eta^{k} \exp (-n \eta)$

in which $a_{m, n}^{k}, b_{m, n}^{k}, c_{m, n}^{k}, d_{m, n}^{k}$ are the coefficients. Based on the rule of solution expressions and the boundary conditions, one can choose the initial guesses $f_{0}, g_{0}, \theta_{0}$, and $\phi_{0}$ as follows:

$f_{0}(\eta)=0$,

$g_{0}(\eta)=\left(1-\alpha_{1}\right)+\left(2 \alpha_{1}-1\right) \exp (-\eta)$,

$\theta_{0}(\eta)=\exp (-\eta)$ $\phi_{0}(\eta)=\exp (-\eta)$

The auxiliary linear operators are

$\mathfrak{£}_{f}=\frac{\mathrm{d}^{3} f}{\mathrm{~d} \eta^{3}}-\frac{\mathrm{d} f}{\mathrm{~d} \eta}$,

$£_{g}=\frac{\mathrm{d}^{2} g}{\mathrm{~d} \eta^{2}}+\frac{\mathrm{d} g}{\mathrm{~d} \eta}$,

$\mathfrak{f}_{\theta}=\frac{\mathrm{d}^{2} \theta}{\mathrm{d} \eta^{2}}-\theta$,

$£_{\varphi}=\frac{\mathrm{d}^{2} \phi}{\mathrm{d} \eta^{2}}-\phi$,

which satisfy

$£_{f}\left[C_{1}+C_{2} \exp (\eta)+C_{3} \exp (-\eta)\right]=0$,

$£_{g}\left[C_{4}+C_{5} \exp (-\eta)\right]=0$,

$£_{\theta}\left[C_{6} \exp (\eta)+C_{7} \exp (-\eta)\right]=0$,

$£_{\phi}\left[C_{8} \exp (\eta)+C_{9} \exp (-\eta)\right]=0$,

where $C_{i}(i=1-9)$ are arbitrary constants.

If $p \in[0,1]$ is an embedding parameter and $\hbar_{f}, \hbar_{g}, \hbar_{\theta}$ and $\hbar_{\phi}$ indicate the non-zero auxiliary parameters, respectively, then the zeroth order deformation problems are

$$
(1-p) \mathfrak{£}_{f}\left[\hat{f}(\eta ; p)-\hat{f}_{0}(\eta)\right]=p \hbar_{f} N_{f}[\hat{f}(\eta ; p), \hat{g}(\eta ; p),
$$$$
\hat{\theta}(\eta ; p), \hat{\phi}(\eta ; p)],
$$

$(1-p) £_{g}\left[\hat{g}(\eta ; p)-\hat{g}_{0}(\eta)\right]=p \hbar_{g} N_{g}[\hat{f}(\eta ; p), \hat{g}(\eta ; p)]$,

$(1-p) \mathfrak{£}_{\theta}\left[\hat{\theta}(\eta ; p)-\hat{\theta}_{0}(\eta)\right]=p \hbar_{\theta} N_{\theta}[\hat{f}(\eta ; p), \hat{\theta}(\eta ; p)]$,

$(1-p) £_{\varphi}\left[\hat{\phi}(\eta ; p)-\hat{\phi}_{0}(\eta)\right]=p \hbar_{\phi} N_{\phi}[\hat{f}(\eta ; p), \hat{\phi}(\eta ; p)]$,

$\hat{f}(0 ; p)=0=\hat{f}^{\prime}(0 ; p), \quad \hat{g}(0 ; p)=\alpha_{1}$,

$\hat{\theta}(0 ; p)=\hat{\phi}(0 ; p)=1$,

$\hat{f}^{\prime}(\infty ; p)=0, \quad \hat{g}(\infty ; p)=1-\alpha_{1}$,

$$
\hat{\theta}(\infty ; p)=\hat{\phi}(\infty ; p)=0,
$$

in which

$N_{f}[\hat{f}(\eta ; p), \hat{g}(\eta ; p), \hat{\theta}(\eta ; p), \hat{\phi}(\eta ; p)]=\frac{\partial^{3} \hat{f}(\eta ; p)}{\partial \eta^{3}}$

$$
\begin{aligned}
& -\hat{f}(\eta ; p) \frac{\partial^{2} \hat{f}(\eta ; p)}{\partial \eta^{2}}+\frac{1}{2}\left(\frac{\partial \hat{f}(\eta ; p)}{\partial \eta}\right)^{2}-M \frac{\partial \hat{f}(\eta ; p)}{\partial \eta} \\
& -2\left[(\hat{g}(\eta ; p))^{2}-\left(1-\alpha_{1}\right)^{2}\right]-2 \lambda_{1}\left(\hat{\theta}(\eta ; p)+N_{1} \hat{\phi}(\eta ; p)\right) \\
& -s\left(\frac{\partial \hat{f}(\eta ; p)}{\partial \eta}+\frac{1}{2} \eta \frac{\partial^{2} \hat{f}(\eta ; p)}{\partial \eta^{2}}\right)
\end{aligned}
$$




$$
\begin{aligned}
& N_{g}[\hat{g}(\eta ; p), \hat{f}(\eta ; p)]=\frac{\partial^{2} \hat{g}(\eta ; p)}{\partial \eta^{2}} \\
&- {\left[\hat{f}(\eta ; p) \frac{\partial \hat{g}(\eta ; p)}{\partial \eta}-\hat{g}(\eta ; p) \frac{\partial \hat{f}(\eta ; p)}{\partial \eta}\right] } \\
&+s\left(1-\alpha_{1}-\hat{g}(\eta ; p)-\frac{1}{2} \eta \frac{\partial \hat{g}(\eta ; p)}{\partial \eta}\right) \\
&\left.-M\left(\hat{g}(\eta ; p)-1+\alpha_{1}\right)\right), \\
& N_{\theta}[\hat{\theta}(\eta ; p), \hat{f}(\eta ; p)]=\frac{1}{\operatorname{Pr}} \frac{\partial^{2} \hat{\theta}(\eta ; p)}{\partial \eta^{2}} \\
&-\left(\hat{f}(\eta ; p) \frac{\partial \hat{\theta}(\eta ; p)}{\partial \eta}-\frac{1}{2} \frac{\partial \hat{f}(\eta ; p)}{\partial \eta} \hat{\theta}(\eta ; p)\right) \\
&-s\left(2 \hat{\theta}(\eta ; p)+\frac{1}{2} \eta \frac{\partial \hat{\theta}(\eta ; p)}{\partial \eta}\right)+\mathrm{Nb} \frac{\partial \hat{\phi}(\eta ; p)}{\partial \eta} \frac{\partial \hat{\theta}(\eta ; p)}{\partial \eta} \\
&+\mathrm{Nt}\left(\frac{\partial \hat{\theta}(\eta ; p)}{\partial \eta}\right)^{2},
\end{aligned}
$$

$N_{\varphi}[\hat{\phi}(\eta ; p), \hat{f}(\eta ; p)]=\frac{\partial^{2} \hat{\phi}(\eta ; p)}{\partial \eta^{2}}$$$
-\operatorname{Le}\left\{\left(\hat{f}(\eta ; p) \frac{\partial \hat{\phi}(\eta ; p)}{\partial \eta}-\frac{1}{2} \frac{\partial \hat{f}(\eta ; p)}{\partial \eta} \hat{\phi}(\eta ; p)\right)\right.
$$$$
\left.-s\left(2 \hat{\varphi}(\eta ; p)+\frac{1}{2} \eta \frac{\partial \hat{\varphi}(\eta ; p)}{\partial \eta}\right)\right\}+\frac{\mathrm{Nt}}{\mathrm{Nb}} \frac{\partial^{2} \hat{\theta}(\eta ; p)}{\partial \eta^{2}} .
$$

For $p=0$ and $p=1$, we have

$\hat{f}(\eta ; 0)=f_{0}(\eta), \quad \hat{f}(\eta ; 1)=f(\eta)$,

$\hat{g}(\eta ; 0)=g_{0}(\eta), \quad \hat{g}(\eta ; 1)=g(\eta)$,

$\hat{\theta}(\eta ; 0)=\theta_{0}(\eta), \quad \hat{\theta}(\eta ; 1)=\theta(\eta)$,

$\hat{\phi}(\eta ; 0)=\varphi_{0}(\eta), \quad \hat{\phi}(\eta ; 1)=\phi(\eta)$.

By Taylor theorem

$$
\begin{aligned}
& \hat{f}(\eta ; p)=f_{0}(\eta)+\sum_{m=1}^{\infty} f_{m}(\eta) p^{m}, \\
& \hat{g}(\eta ; p)=g_{0}(\eta)+\sum_{m=1}^{\infty} g_{m}(\eta) p^{m}, \\
& \hat{\theta}(\eta ; p)=\theta_{0}(\eta)+\sum_{m=1}^{\infty} \theta_{m}(\eta) p^{m}, \\
& \hat{\phi}(\eta ; p)=\phi_{0}(\eta)+\sum_{m=1}^{\infty} \phi_{m}(\eta) p^{m},
\end{aligned}
$$

$$
\begin{aligned}
& f_{m}(\eta)=\left.\frac{1}{m !} \frac{\partial^{m} f(\eta ; p)}{\partial \eta^{m}}\right|_{p=0}, g_{m}(\eta)=\left.\frac{1}{m !} \frac{\partial^{m} g(\eta ; p)}{\partial \eta^{m}}\right|_{p=0}, \\
& \theta_{m}(\eta)=\left.\frac{1}{m !} \frac{\partial^{m} \theta(\eta ; p)}{\partial \eta^{m}}\right|_{p=0}, \phi_{m}(\eta)=\left.\frac{1}{m !} \frac{\partial^{m} \phi(\eta ; p)}{\partial \eta^{m}}\right|_{p=0}
\end{aligned}
$$

and

$$
\begin{aligned}
& f(\eta)=f_{0}(\eta)+\sum_{m=1}^{\infty} f_{m}(\eta), \\
& g(\eta)=g_{0}(\eta)+\sum_{m=1}^{\infty} g_{m}(\eta), \\
& \theta(\eta)=\theta_{0}(\eta)+\sum_{m=1}^{\infty} \theta_{m}(\eta), \\
& \phi(\eta)=\phi_{0}(\eta)+\sum_{m=1}^{\infty} \phi_{m}(\eta) .
\end{aligned}
$$

The $m$ th-order deformation problems are defined as

$$
\begin{aligned}
& £_{f}\left[f_{m}(\eta)-\chi_{m} f_{m-1}(\eta)\right]=\hbar_{f} R_{m}^{f}(\eta), \\
& £_{g}\left[g_{m}(\eta)-\chi_{m} g_{m-1}(\eta)\right]=\hbar_{g} R_{m}^{g}(\eta), \\
& £_{\theta}\left[\theta_{m}(\eta)-\chi_{m} \theta_{m-1}(\eta)\right]=\hbar_{\theta} R_{m}^{\theta}(\eta), \\
& £_{\phi}\left[\phi_{m}(\eta)-\chi_{m} \phi_{m-1}(\eta)\right]=\hbar_{\phi} R_{m}^{\phi}(\eta), \\
& f_{m}(0)=f_{m}^{\prime}(0)=g_{m}(0)=\theta_{m}(0)=\varphi_{m}(0)=0, \\
& f_{m}^{\prime}(\infty)=g_{m}(\infty)=\theta_{m}(\infty)=\varphi_{m}(\infty)=0,
\end{aligned}
$$

where

$$
\begin{aligned}
R_{m}^{f}(\eta) & =f_{m-1}^{\prime \prime \prime}-\sum_{k=0}^{m-1} f_{k} f_{m-1-k}^{\prime \prime}+\frac{1}{2} \sum_{k=0}^{m-1} f_{k}^{\prime} f_{m-1-k}^{\prime} \\
& -2\left[\sum_{k=0}^{m-1} g_{k} g_{m-1-k}-\left(1-\alpha_{1}\right)^{2}\left(1-\chi_{m}\right)\right] \\
& -2 \lambda_{1}\left(\theta_{m-1}+N_{1} \phi_{m-1}\right)-s\left(f_{m-1}^{\prime}+\frac{1}{2} \eta f_{m-1}^{\prime \prime \prime}\right)-M f_{m-1}^{\prime},
\end{aligned}
$$

$$
\begin{aligned}
R_{m}^{g}(\eta)= & g_{m-1}^{\prime \prime}-\sum_{k=0}^{m-1}\left[f_{k} g_{m-1-k}^{\prime}-g_{k} f_{m-1-k}^{\prime}\right] \\
& +s\left[\left(1-\alpha_{1}\right)\left(1-\chi_{m}\right)-g_{m-1}-\frac{1}{2} \eta g_{m-1}^{\prime}\right] \\
& -M\left(g_{m-1}-1-\alpha_{1}\right)
\end{aligned}
$$




$$
\begin{aligned}
& R_{m}^{\theta}(\eta)= \frac{1}{\operatorname{Pr}} \theta_{m-1}^{\prime \prime}-\sum_{k=0}^{m-1}\left[f_{k} \theta_{m-1-k}^{\prime}-\frac{1}{2} \theta_{k} f_{m-1-k}^{\prime}\right] \\
&-s\left(2 \theta_{m-1}+\frac{1}{2} \eta \theta_{m-1}^{\prime}\right)+\mathrm{Nb} \sum_{k=0}^{m-1} \phi_{k}^{\prime} \theta_{m-1-k}^{\prime} \\
&+\mathrm{Nt} \sum_{k=0}^{m-1} \theta_{k}^{\prime} \theta_{m-1-k}^{\prime}, \\
& R_{m}^{\phi}(\eta)= \phi_{m-1}^{\prime \prime}-\mathrm{Le}\left\{\sum_{k=0}^{m-1}\left[f_{k} \phi_{m-1-k}^{\prime}-\frac{1}{2} \phi_{k} f_{m-1-k}^{\prime}\right]\right. \\
&-s\left(\begin{array}{ll}
2 \phi_{m-1} \\
\left.\left.+\frac{1}{2} \eta \phi_{m-1}^{\prime}\right)\right\}+\frac{\mathrm{Nt}}{\mathrm{Nb}} \theta_{m-1}^{\prime \prime},
\end{array}\right. \\
& \chi_{m}= \begin{cases}0 & m \leq 1, \\
1 & m>1 .\end{cases}
\end{aligned}
$$

The general solution of Eqs. (58)-(61) can be written as

$$
\begin{aligned}
& f_{m}(\eta)=f_{m}^{*}(\eta)+C_{1}+C_{2} \exp (\eta)+C_{3} \exp (-\eta), \\
& g_{m}(\eta)=g_{m}^{*}(\eta)+C_{4}+C_{5} \exp (-\eta), \\
& \theta_{m}(\eta)=\theta_{m}^{*}(\eta)+C_{6} \exp (\eta)+C_{7} \exp (-\eta), \\
& \phi_{m}(\eta)=\phi_{m}^{*}(\eta)+C_{8} \exp (\eta)+C_{9} \exp (-\eta),
\end{aligned}
$$

where $f_{m}^{*}(\eta), g_{m}^{*}(\eta), \theta_{m}^{*}(\eta)$ and $\phi_{m}^{*}(\eta)$ are the special solutions.

The numerical data of these solutions have been computed and presented through graphs and tables.

\section{Convergence of the homotopy solutions}

Analytical solutions to the governing ordinary differential Eqs. (6)-(9) with boundary conditions (10) are obtained using HAM. The series solutions depend on the non-zero auxiliary parameters $\hbar_{f}, \hbar_{g}, \hbar_{\theta}$ and $\hbar_{\phi}$ which can adjust and

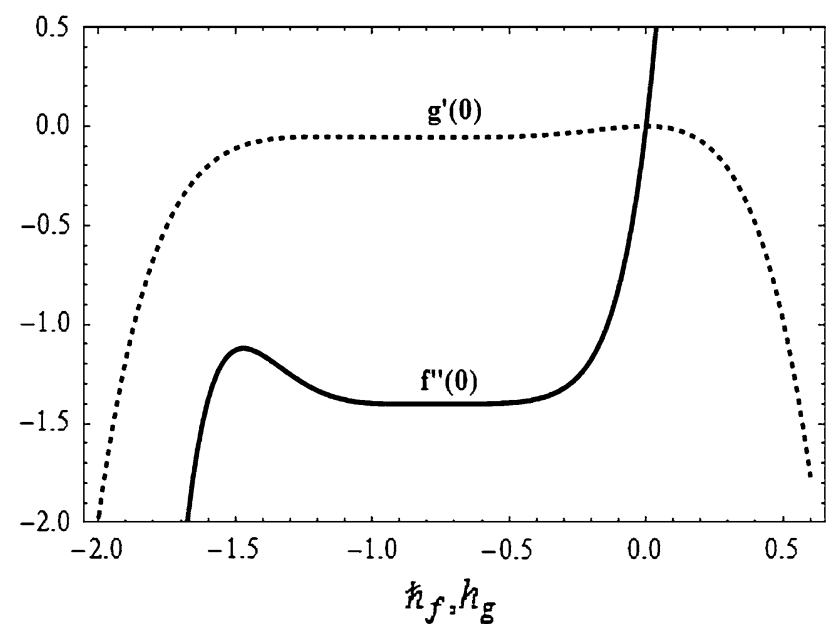

Fig. $2 \hbar$-Curve of $f^{\prime \prime}(0)$ and $g^{\prime}(0)$ at 10th approximation (PWT case)

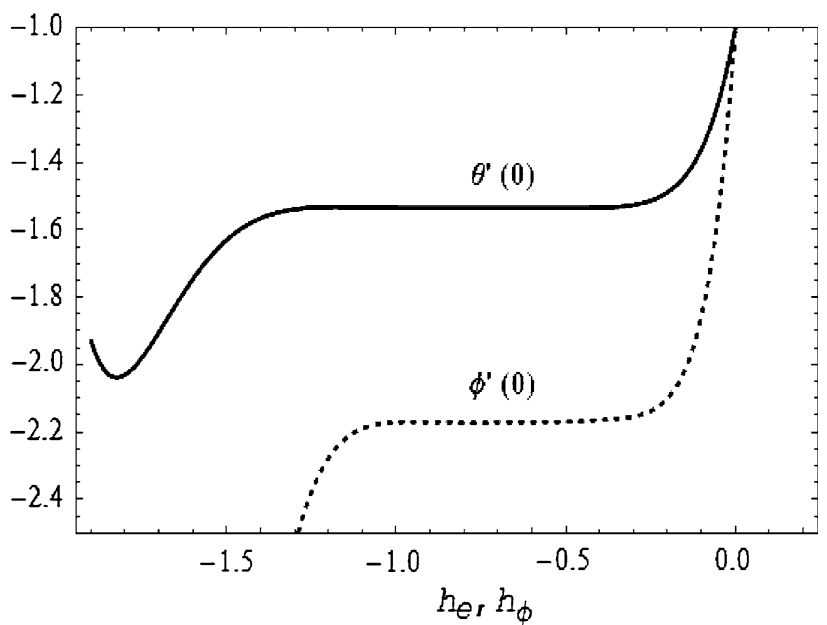

Fig. $3 \hbar$-Curve for $\theta^{\prime}(0)$ and $\varphi^{\prime}(0)$ at 10 th approximation (PWT case)

Table 1 Convergence of HAM solution for different order of approximations

\begin{tabular}{lllll}
\hline $\begin{array}{l}\text { Order of } \\
\text { convergence }\end{array}$ & $-f^{\prime \prime}(0)$ & $-g^{\prime}(0)$ & $-\theta^{\prime}(0)$ & $-\phi^{\prime}(0)$ \\
\hline 2 & 1.3618 & 0.025 & 1.51935 & 1.31471 \\
5 & 1.55294 & 0.0637 & 1.55335 & 1.25495 \\
10 & 1.55632 & 0.0669 & 1.55332 & 1.25182 \\
15 & 1.55621 & 0.0666 & 1.55325 & 1.25182 \\
20 & 1.55621 & 0.0664 & 1.55325 & 1.25182 \\
25 & 1.55621 & 0.0664 & 1.55325 & 1.25182 \\
30 & 1.55621 & 0.0664 & 1.55325 & 1.25182 \\
\hline
\end{tabular}

control the convergence of the HAM solutions. The range of admissible values of auxiliary parameters is seen by plotting $\hbar$ - curve of the functions $f^{\prime \prime}(0), g^{\prime}(0), \theta^{\prime}(0)$ and $\phi^{\prime}(0)$ for 10-order of approximations in Figs. 2 and 3. It is found that the range of permissible values of $\hbar_{f}, \hbar_{g}, \hbar_{\theta}$ and $\hbar_{\phi} \quad$ is $\quad-1.1 \leq \hbar_{f} \leq-0.4, \quad-1.1 \leq \hbar_{g} \leq-0.5, \quad-1.3 \leq$ $\hbar_{\theta} \leq-0.4$, and $-1.0 \leq \hbar_{\phi} \leq-0.4$. Also, our computation shows that the series solution converges in the whole region of $\eta$ when $\hbar_{f}=\hbar_{g}=\hbar_{\theta}=\hbar_{\phi}=-0.8$.

The convergence (Table 1) is prepared for each of the function up to 30th order of approximation. It is found that the convergence is achieved up to 15 th order of approximation.

\section{Results and discussion}

The main focus of this section is to observe the effects of emerging parameters on the velocity, temperature and concentration fields for PWT case. The effects of variations are depicted in Figs. 4, 5, 6, 7, 8, 9, 10, 11, 12, 13 and 14. 


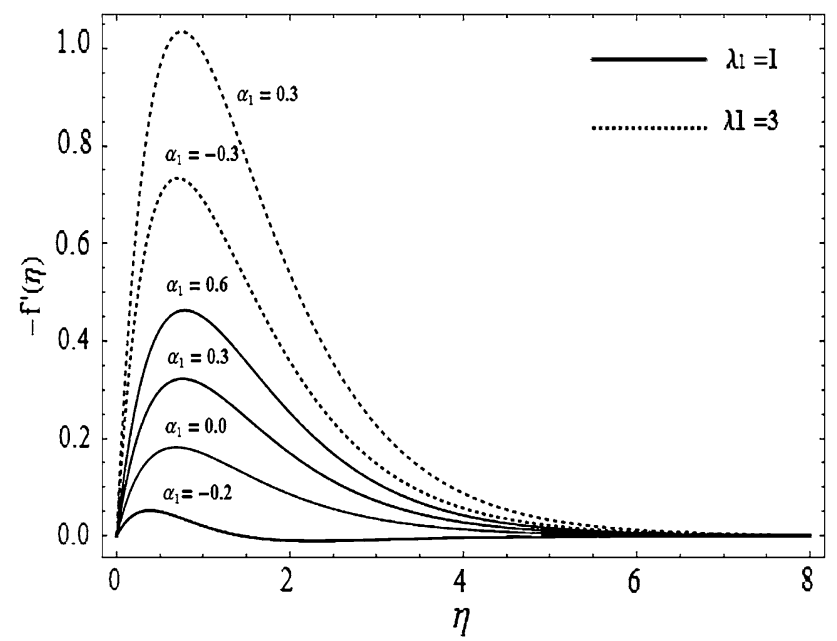

Fig. 4 Variation of $\alpha_{1}$ and $\lambda_{1}$ on $-f$ when $N=1, \operatorname{Pr}=0.7$, $\mathrm{Nb}=0.1, \mathrm{Nt}=0.1, \mathrm{Le}=4, M=1, s=0.5$

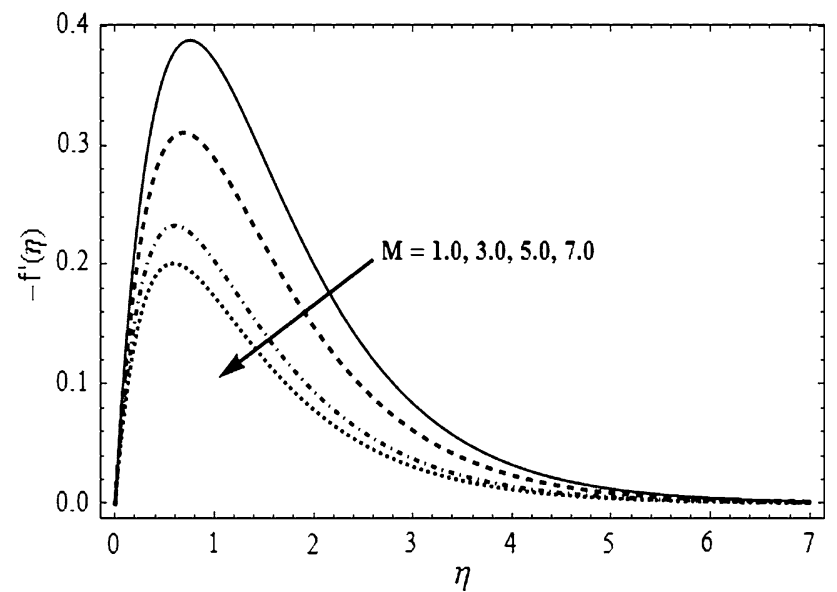

Fig. 5 Variation of $M$ on $-f^{\prime}$ when $\lambda_{1}=1, \alpha_{1}=0.6 \quad N=1$, $\mathrm{Pr}=0.7, \mathrm{Nb}=0.1, \mathrm{Nt}=0.1, \mathrm{Le}=4, s=0.5$

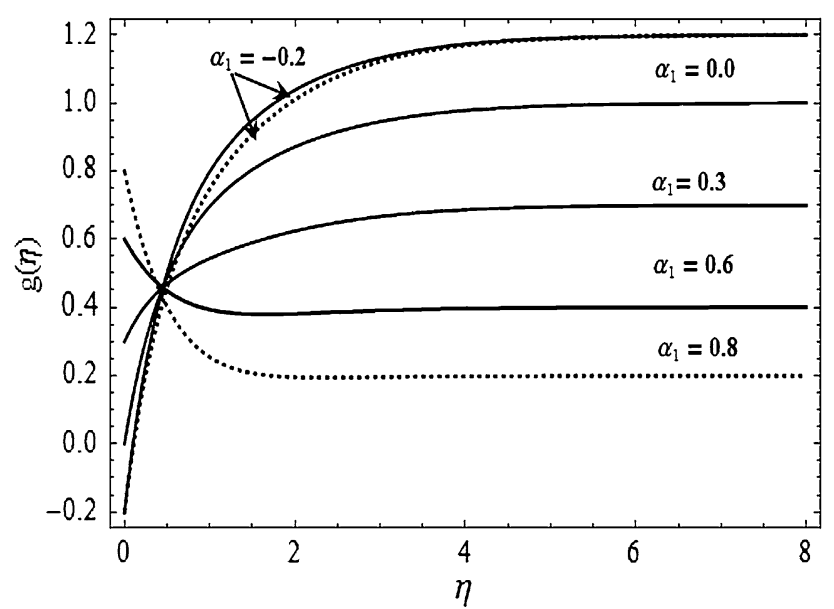

Fig. 6 Variation of $\alpha_{1}$ and $\lambda_{1}$ on $g$ when $N=1, \quad \operatorname{Pr}=0.7$, $\mathrm{Nb}=0.1, \mathrm{Nt}=0.1, \mathrm{Le}=4, M=1, s=0.5$

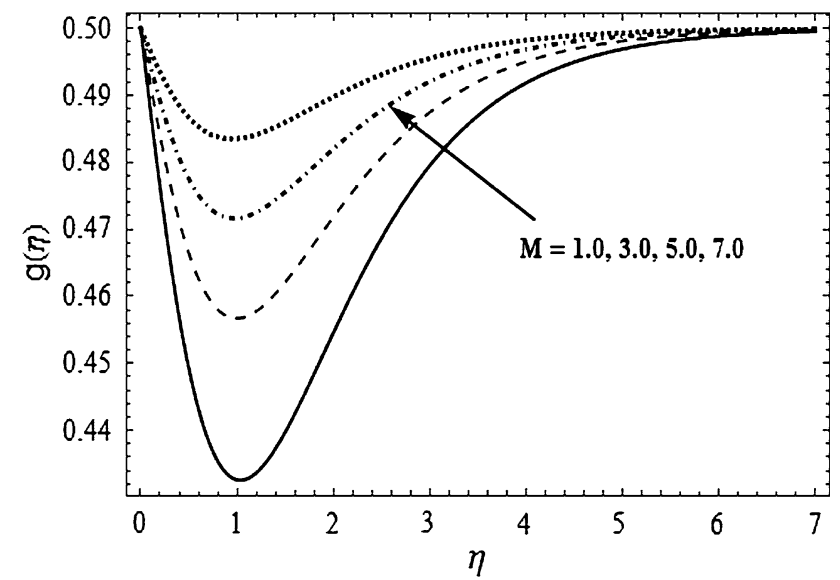

Fig. 7 Variation of $M$ on $g$ when $\lambda_{1}=1, \alpha_{1}=0.6, \quad N=1$, $\mathrm{Pr}=0.7, \mathrm{Nb}=0.1, \mathrm{Nt}=0.1, \quad \mathrm{Le}=4, s=0.5$

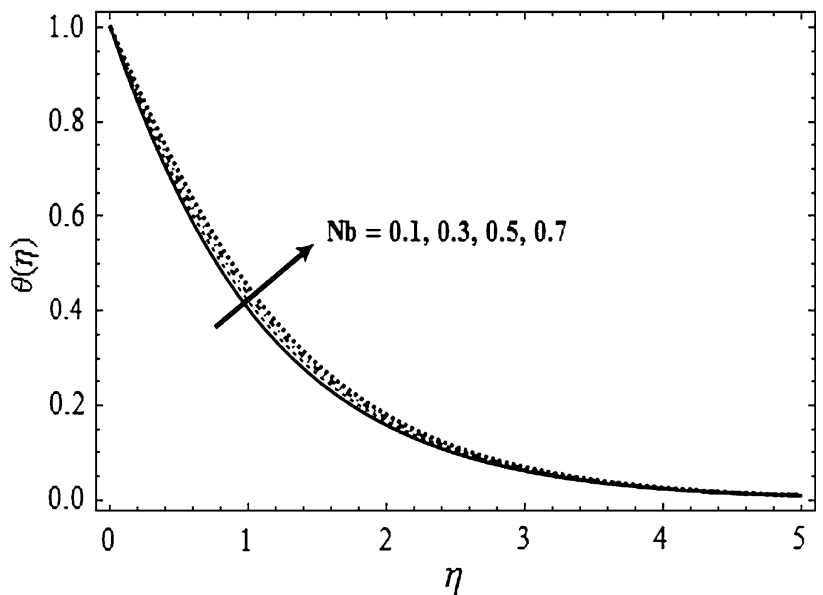

Fig. 8 Variation of Brownian motion parameter $\mathrm{Nb}$ on temperature $\theta$ when $\lambda_{1}=1, \quad \alpha_{1}=0.6, \quad N=1, \operatorname{Pr}=0.7, M=1, \quad \mathrm{Nt}=0.1$, Le $=4, s=0.5$

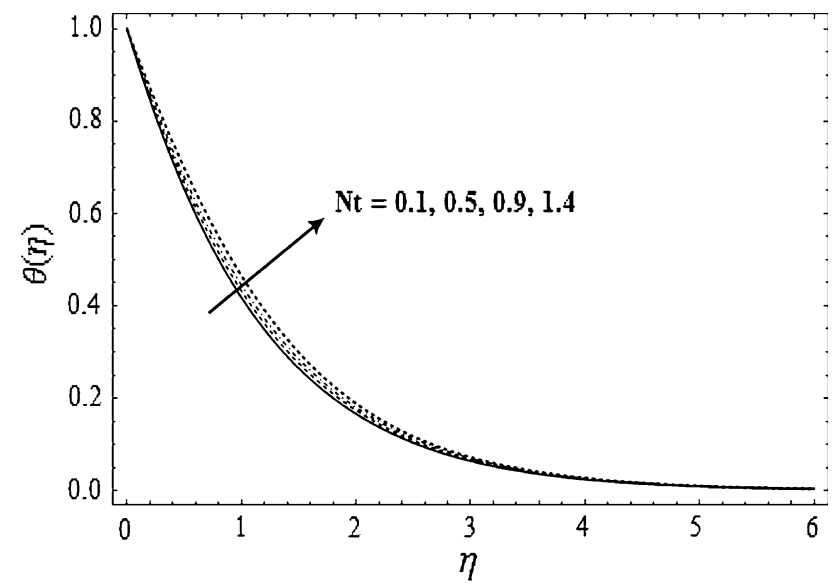

Fig. 9 Variation of thermophoresis parameter $\mathrm{Nt}$ on temperature $\theta$ when $\lambda_{1}=1, \alpha_{1}=0.6, \quad N=1, \quad \operatorname{Pr}=0.7, \quad \mathrm{Nb}=0.1, \quad M=1$, Le $=4, s=0.5$ 


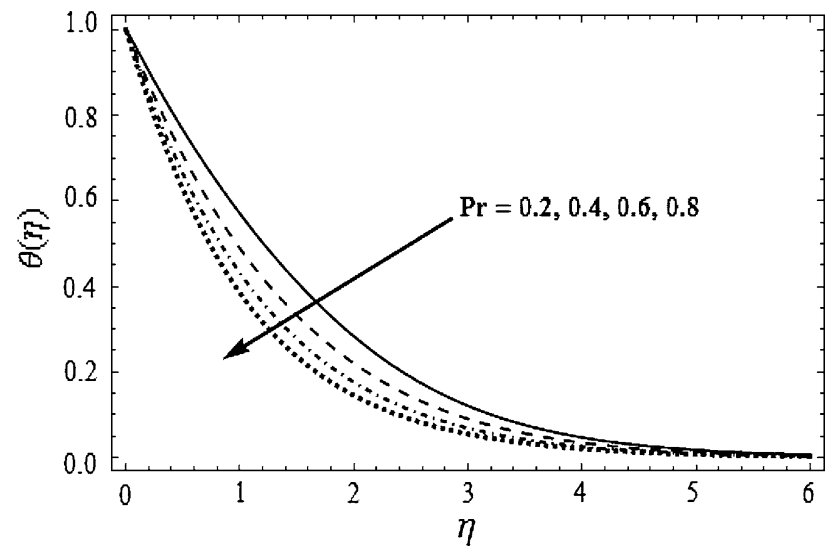

Fig. 10 Variation of Prandtl number $\operatorname{Pr}$ on temperature $\theta$ when $\lambda_{1}=1, \quad \alpha_{1}=0.6, \quad N=1, \quad M=1, \quad \mathrm{Nb}=0.1, \quad \mathrm{Nt}=0.1$, Le $=4, s=0.5$

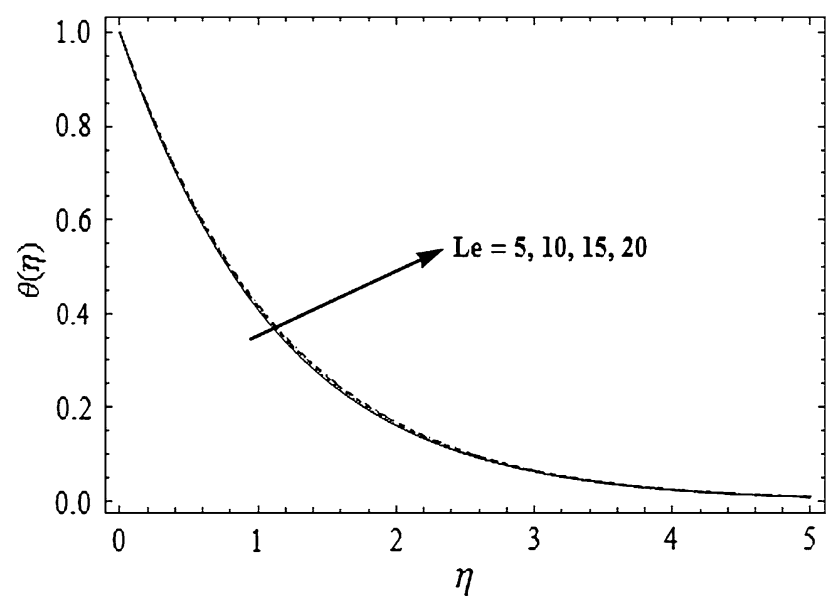

Fig. 11 Variation of Lewis number Le on temperature $\theta$ when $\lambda_{1}=1, \alpha_{1}=0.6, N=1, \quad \operatorname{Pr}=0.7, \mathrm{Nb}=0.1, \mathrm{Nt}=0.1, M=1$, $s=0.5$

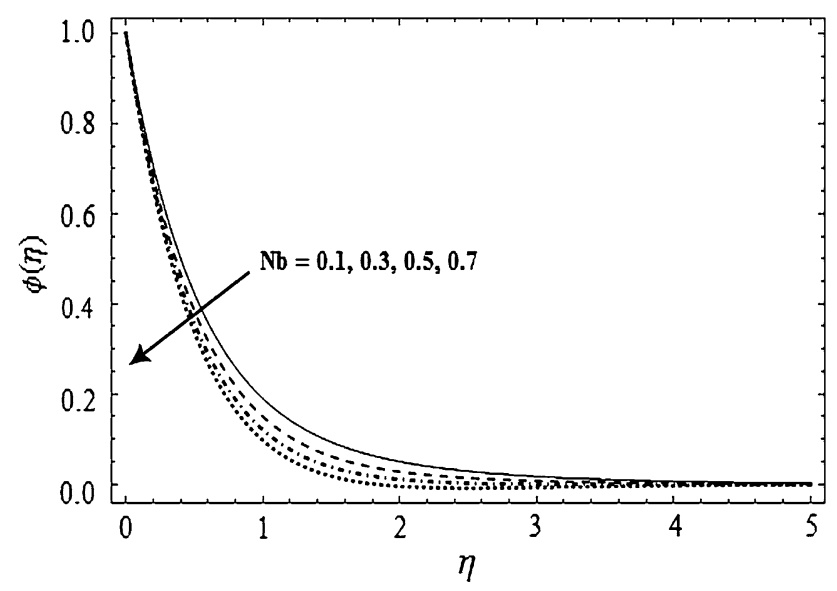

Fig. 12 Variation of thermophoresis parameter $\mathrm{Nb}$ on concentration $\phi$ when $\lambda_{1}=1, \alpha_{1}=0.6, N=1, \operatorname{Pr}=0.7, M=1, \mathrm{Nt}=0.1$, Le $=4, s=0.5$

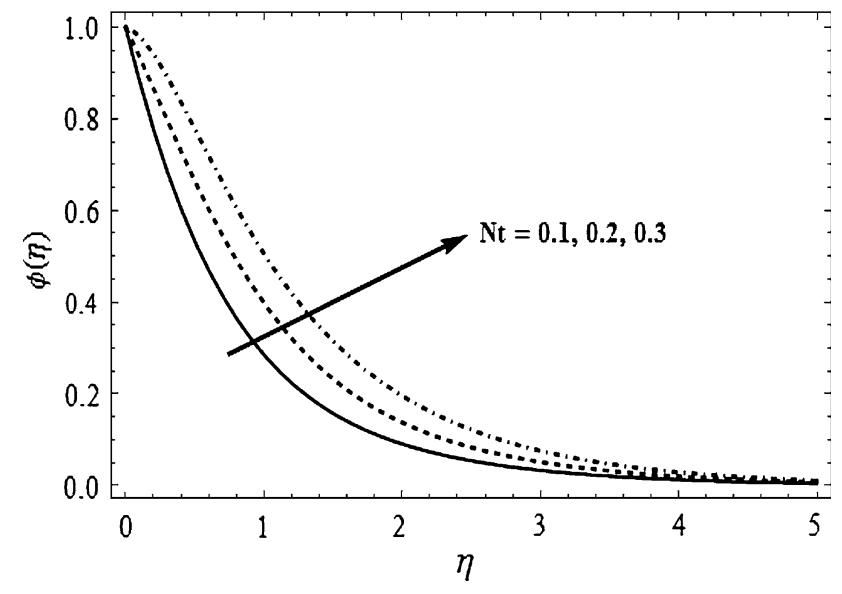

Fig. 13 Variation of thermophoresis parameter $\mathrm{Nt}$ on concentration $\phi$ when $\lambda_{1}=1, \alpha_{1}=0.6, N=1, \operatorname{Pr}=0.7, \mathrm{Nb}=0.1, M=1$, Le $=4, s=0.5$

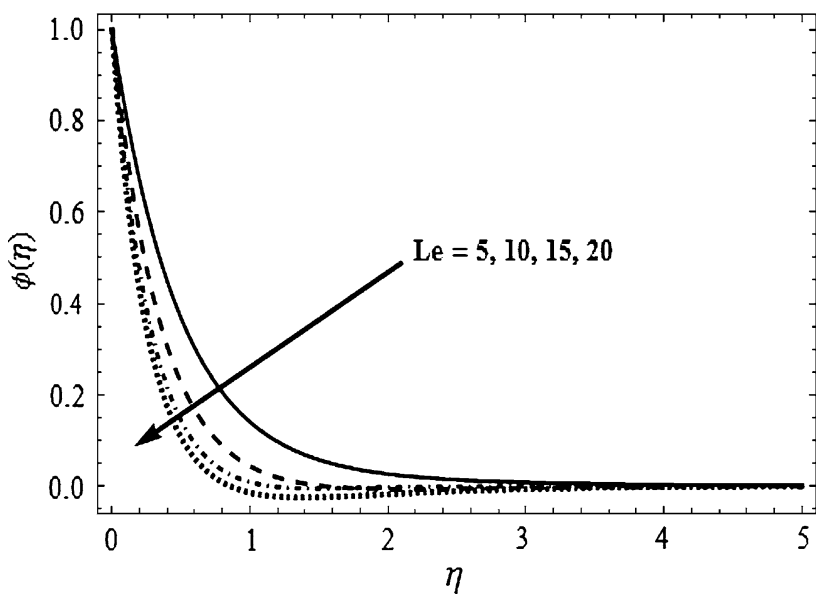

Fig. 14 Variation of Lewis number Le on concentration $\phi$ when $\lambda_{1}=1, \quad \alpha_{1}=0.6, \quad N=1, \quad \operatorname{Pr}=0.7, \quad \mathrm{Nb}=0.1, \quad \mathrm{Nt}=0.1$, $M=1, s=0.5$

The variation of tangential velocity $-f^{\prime}$ for combined effects of $\alpha_{1}$ and $\lambda_{1}$ is plotted in Fig. 4. The fluid and the cone are rotating with equal angular velocity in the same direction for $\alpha_{1}=0.5$. The positive Buoyancy force (i.e., $\lambda_{1}=1$ ), which behaves as favorable pressure gradient, is responsible for the flow. For $\alpha_{1}>0.5$, the velocity $-f^{\prime}$ increases its magnitude; further the behavior is opposite when $\alpha_{1}<0.5$. The velocity $-f^{\prime}$ decreases with the increase in $M$, whereas the boundary layer thickness decreases (see Fig. 5). Thus, we say that magnetic field causes the reduction of boundary layer. In Fig. 6, it is seen that with the increase of $\alpha_{1}$, azimuthal velocity $g(\eta)$ decreases for $\alpha_{1}>0.5$, but the behavior is opposite for $\alpha_{1}<0.5$. Figure 7 illustrates that both the velocity and boundary layer thickness increase with the increasing $M$. The variation of $\mathrm{Nb}$ on temperature profile is plotted in Fig. 8. It is clear from the figure that with an increase in 
Table 2 Comparison of the results $\left[-f^{\prime \prime}(0),-g^{\prime}(0),-\theta^{\prime}(0)\right]$ with those of Anilkumar and Roy (2004)

\begin{tabular}{|c|c|c|c|c|c|c|c|}
\hline \multirow[t]{2}{*}{$\lambda_{1}$} & \multirow[t]{2}{*}{$\alpha_{1}$} & \multicolumn{3}{|c|}{ Present results } & \multicolumn{3}{|c|}{ Numerical results (Anilkumar and Roy 2004) } \\
\hline & & $-f^{\prime \prime}(0)$ & $-g^{\prime}(0)$ & $-\theta^{\prime \prime}(0)$ & $-f^{\prime \prime}(0)$ & $-g^{\prime}(0)$ & $-\theta^{\prime}(0)$ \\
\hline \multirow[t]{4}{*}{1} & 0 & 0.63243 & -0.63948 & 0.81920 & 0.63241 & -0.63949 & 0.81922 \\
\hline & 0.25 & 1.31337 & -0.22764 & 0.89010 & 1.31339 & -0.22765 & 0.89011 \\
\hline & 0.50 & 1.84795 & 0.19805 & 0.93706 & 1.84798 & 0.19806 & 0.93700 \\
\hline & 0.75 & 2.24658 & 0.62679 & 0.96560 & 2.24659 & 0.62679 & 0.96563 \\
\hline \multirow[t]{4}{*}{3} & 0 & 3.79524 & -0.59651 & 1.02869 & 3.79522 & -0.59651 & 1.02869 \\
\hline & 0.25 & 4.31853 & -0.13694 & 1.06538 & 4.31854 & -0.13691 & 1.06539 \\
\hline & 0.50 & 4.73959 & 0.33553 & 1.09111 & 4.73958 & 0.33552 & 1.09111 \\
\hline & 0.75 & 5.05950 & 0.81200 & 1.10711 & 5.05951 & 0.81201 & 1.10712 \\
\hline
\end{tabular}

Table 3 Values of skin-friction coefficients for different parameters

\begin{tabular}{ccccc}
\hline$\lambda_{1}$ & $N$ & $M$ & $C_{f x} R e_{x}^{1 / 2}$ & $C_{f x} R e_{x}^{1 / 2}$ \\
\hline 1 & 1 & 1 & 1.93701 & 0.352519 \\
2 & & & 3.53193 & 0.425810 \\
3 & & 5.14716 & 0.556833 \\
4 & & & 6.57918 & 0.651836 \\
& & & 0.430112 & 0.023773 \\
& 0.5 & & 0.888909 & 0.049316 \\
& 0.5 & & 1.34049 & 0.074587 \\
& 1 & & 1.78487 & 0.099586 \\
& & 0 & 1.7251 & 0.10712 \\
& & 1 & 1.5355 & 0.08695 \\
& & 1.5 & 1.4552 & 0.07787 \\
& & 2 & 1.3836 & 0.06944 \\
\hline
\end{tabular}

$\mathrm{Nb}$, the temperature increases. The effects of $\mathrm{Nt}$ on temperature profile are seen in Fig. 9. It is seen that temperature profile increases with the increase in Nt. Figure 10 explains the effects of $\operatorname{Pr}$ on temperature profile $\theta$. It is found that thermal boundary layer thickness decreases with the increasing Pr. Temperature profile has a small change with an increase in Le (see Fig. 11). It is depicted from Fig. 12 that concentration profile decreases with the increasing $\mathrm{Nb}$. However, with increase in $\mathrm{Nt}$, concentration profile increases and th layer thickness reduces (see Fig. 13). The behavior of Le on concentration profile is plotted in Fig. 14. It is inferred that concentration profile decreases as Le increase. It is depicted that our series solutions are in good agreement with the numerical results reported by Anilkumar and Roy (2004) in the absence of $\mathrm{Nb}, \mathrm{Nt}$ and $M$ (see Table 2). The skin-friction coefficients are an increasing function of $\lambda_{1}$, and $N$. On the other hand, the variation is opposite for $M$ (see Table 3). Table 4 provides the numerical values of Nusselt number $-\theta^{\prime}(0)$ and Sherwood number $-\phi^{\prime}(0)$ for various values of $\mathrm{Nb}, \mathrm{Nt}$, $\operatorname{Pr}$ and Le, respectively. From Table 4, it is obvious that the
Table 4 Values of Nusselt number and Sherwood number for different pertinent parameters

\begin{tabular}{llllll}
\hline $\mathrm{Nb}$ & $\mathrm{Nt}$ & $\mathrm{Pr}$ & $\mathrm{Le}$ & $\mathrm{Nu} R e_{x}^{-1 / 2}$ & $\mathrm{Sh} R e_{x}^{-1 / 2}$ \\
\hline 0.1 & 0.1 & 0.7 & 4 & 0.847015 & 1.87446 \\
0.2 & & & & 0.813260 & 1.98863 \\
0.3 & & & & 0.782095 & 2.02702 \\
0.4 & & & & 0.752381 & 2.04649 \\
& 0.2 & & & 0.834721 & 1.68770 \\
& 0.3 & & & 0.822616 & 1.51714 \\
& 0.4 & & & 0.810698 & 1.36180 \\
& 0.5 & & & 0.798960 & 1.22074 \\
& 0.4 & & 0.969840 & 1.28445 \\
& 0.8 & & 1.269210 & 1.26374 \\
& 1.2 & & 1.509040 & 1.25355 \\
& 1.6 & & 1.694130 & 1.24753 \\
& & 4 & 0.838055 & 1.88033 \\
& & 8 & 0.823504 & 2.70267 \\
& & 12 & 0.813247 & 3.43456 \\
& & 16 & 0.785512 & 3.81396 \\
\hline
\end{tabular}

Nusselt number $-\theta^{\prime}(0)$ decreases with the increasing $\mathrm{Nb}$, $\mathrm{Nt}$, and Le. However, increases by an increase in Pr. Further we have seen that the Sherwood number $-\phi^{\prime}(0)$ have an increasing behavior with an increase in $\mathrm{Nb}$ and Le but decreases with an increase in Nt and Pr. Since the equations for both PWT case and PHF case have almost the same structure, the results for PHF case are ignored.

\section{Concluding remarks}

Unsteady mixed convection flow of a rotating nanofluid over a rotating cone is studied in the presence of magnetic field. The solutions are carried out by a well-known analytical method HAM. The velocity $-f^{\prime}(\eta)$ is an increasing function of $\alpha_{1}$. The behavior of $M$ on velocities 
$\left(-f^{\prime}(\eta)^{\prime}, g(\eta)\right)$ is just opposite. The effect of $\operatorname{Pr}$ is to decrease the temperature profile $\theta$. The temperature profile $\theta$ increases as $\mathrm{Nb}, \mathrm{Nt}$ and Le increase. The influence of $\mathrm{Nb}$ and $\mathrm{Nt}$ are opposite for concentration field $\phi$. The concentration field $\phi$ decreases with the increasing Le. Skinfriction coefficients show an increasing behavior with an increase in the ratio of buoyancy force $N$. Our present results are in good agreement with the previous results available in Anilkumar and Roy (2004).

Open Access This article is distributed under the terms of the Creative Commons Attribution License which permits any use, distribution, and reproduction in any medium, provided the original author(s) and the source are credited.

\section{References}

Abbasbandy S (2006) Approximate solution of the nonlinear model of diffusion and reaction catalysts by means of the homotopy analysis method. Chem Eng 136:144-150

Abbasbandy S (2008) The application of homotopy analysis method to nonlinear equations arising in heat transfer. Phys Lett A 360:109-113

Abbasbandy S, Samadian F (2008) Soliton solutions for the 5th-order $\mathrm{KdV}$ equation with the homotopy analysis method. Nonlinear Dyn 51:83-87

Aldoss TK (1996) MHD mixed convection from a vertical cylinder embedded in a porous medium. Int Commun Heat Mass Trans 23:517-530

Anilkumar D, Roy S (2004) Unsteady mixed convection flow on a rotating cone in a rotating fluid. Appl Math Comput 155:545-561

Bachok N, Ishak A, Pop I (2010) Boundary Layer flow of nanofluid over a moving surface in a flowing fluid. Int $\mathbf{J}$ Therm Sci 49:1663-1668

Bararnia H, Abdoul R, Ghotbi, Domairry G (2009) On the analytical solution for MHD natural convection flow and heat generation fluid in porous medium. Comm Nonlinear Sci Numer Simul 14:2689-2701

Buongiorno J (2006) Convective transport in nanofluids. ASME J Heat Trans 128:240-250

Duangthongsuk W, Wongwises S (2007) A critical review of convective heat transfer nanofluids. Renew Sustain Energy Rev 11:797-817

Duangthongsuk W, Wongwises S (2008) Effect of thermophysical properties models on the predicting of the convective heat transfer coefficient for low concentration nanofluid. Int Commun Heat Mass Transf 35:1320-1326

Ellahi R, Afzal S (2009) Effects of variable viscosity in a third grade fluid with porous medium: an analytic solution. Commun Nonlinear Sci Numer Simul 14:2056-2072

Ellahi R, Riaz A (2010) Analytical solutions for MHD flow in a thirdgrade fluid with variable viscosity. Math Comput Modell 52:1783-1793
Hering RG, Grosh RJ (1963) Laminar combined convection from a rotating cone. ASME J Heat Trans 85:29-34

Himasekhar K, Sarma PK, Janardhan K (1989) Laminar mixed convection from a vertical rotating cone. Int Commun Heat Mass Trans 16:99-106

Hojjat M, Etemad SG, Bagheri R (2010) Laminar heat transfer of nanofluid in a circular tube. Korean J Chem Eng 27:1391-1396

Ishak A, Nazar R, Bachok N, Pop I (2010) MHD mixed convection flow near the stagnation-point on a vertical permeable surface. Physica A Stat Mech Appl 389:40-46

Kakaç S, Pramuanjaroenkij A (2009) Review of convective heat transfer enhancement with nanofluids. Int J Heat Mass Trans 52:3187-3196

Kakarantzas SC (2009) Magnetohydrodynamic natural convection in a vertical cylindrical cavity with sinusoidal upper wall temperature. Int J Heat Mass Trans 52:250-259

Khan WA, Pop I (2010) Boundary-layer flow of a nanofluid past a stretching sheet. Int. J Heat Mass Trans 53:2477-2483

Liao SJ(2003) Beyond perturbation: introduction to the homotopy analysis method. Chapman \& Hall/CRC Press, Boca Raton

Liao SJ (2004)On the homotopy analysis method for nonlinear problems. Appl Math Comput 147:499-513

Liao SJ (2005) Comparison between the homotopy analysis method and homotopy perturbation method. Appl Math Comput 169:1186-1194

Liao SJ (2009) Notes on the homotopy analysis method: some definitions and theorems. Commun Nonlinear Sci Numer Simulat 14:983-997

Liao SJ, Cheung kf (2003) Homotopy analysis of nonlinear progressive waves in deep water. J Eng Math 145:105-116

Lin HT, Lin LK (1987) Heat transfer from a rotating cone or disk to fluids of any prandtl number. Int Commun Heat Mass Trans $14: 323-332$

Makinde OD, Aziz A (2011) Boundary layer flow of a nano fluid past a stretching sheet with a convective boundary condition. Int $\mathbf{J}$ Therm Sci 50:1326-1332

Nadeem S, Haq RU, (2012) MHD boundary layer flow of a nanofuid past a porous shrinking sheet with thermal radiation. J Aerospace Eng. doi:10.1061(ASCE)AS.1943-5525:0000299

Nadeem S, Hussain A (2009) MHD flow of a viscous fluid on a nonlinear porous shrinking sheet with HAM. Appl Math Mech Engl Ed 30:1-10

Nadeem S, Lee C (2012) Boudary layer flow of nanofluid over an exponentially stretching sheet. Nanoscale Res Lett 94:7

Nadeem S, Saleem S (2013) Analytical treatment of unsteady mixed convection MHD flow on a rotating cone in a rotating frame. J Taiwan Inst Chem Eng. doi:10.1016/j.jtice.2013.01.007

Nadeem S, Hussain A, Khan M (2010) HAM solutions for boundary layer flow in the region of the stagnation point towards a stretching sheet. Commun Nonlinear Sci NumerSimul $15: 475-481$

Tien CL (1960) Heat transfer by laminar flow from a rotating cone. ASME J Heat Trans 82:252-253

Wang TY, kleinstreur C (1990) Similarity solutions of combined convection heat transfer from a rotating cone or disk to nonNewtonian fluids. ASME J Heat Trans 112:939-944 